\title{
LA PROTECCIÓN POR MATERNIDAD DE LAS ROFESIONALES COLEGIADAS: DIFERENCIAS ENTRE AUTÓNOMAS INTEGRADAS EN EL RETA O EN UNA MUTUALIDAD ALTERNATIVA
}

MATERNITY PROTECTION OF PROFESSIONALS COLLEGIATE: REGIME APPLICABLE TO THOSE INTEGRATED IN RETA AND THOSE WHO ARE INCLUDED IN AN ALTERNATIVE MUTUALITY

\section{BELÉN GARCÍA ROMERO}

Profesora Titular de Derecho del Trabajo y Seguridad Social

Universidad de Murcia 


\title{
RESUMEN
}

El presente trabajo estudia el régimen jurídico de la prestación por maternidad biológica de las trabajadoras autónomas, en especial, de las profesionales liberales colegiadas incluidas en una Mutualidad de Previsión Social alternativa al RETA. Con ello se ponen de relieve las diferencias en materia de derechos y obligaciones de uno y otro colectivo de trabajadoras por cuenta propia, así como la reducida aplicabilidad del derecho de cesión al padre del disfrute del período de descanso por maternidad en el supuesto de profesionales colegiadas, tras la fijación de un nivel mínimo de cobertura y prestaciones por la Ley 27/2011. En particular, se analiza el modo en que las Mutualidades Alternativas al RETA cumplen con las exigencias legales de asegurar una protección mínima frente a determinadas contingencias, entre las que se encuentra la de maternidad, y si ello vacía o no de contenido la previsión relativa al derecho de cesión a favor del padre.

PALABRAS CLAVE: trabajadoras autónomas, prestación de maternidad, Régimen Especial de la Seguridad Social, Mutualidades.

\begin{abstract}
This paper studies the legal status of biological maternity allowance for self-employed, especially of liberal professionals collegiate included in a Social Security Mutual alternative to RETA. This highlights the differences in the rights and obligations of both collective of self-employed, as well as the reduced applicability of the right to transfer to the father of the enjoyment of the period of maternity leave in the course of professional collegiate after setting a minimum level of coverage and benefits by Law 27/2011. In particular, it is analysed the way that the Alternatives Mutual to RETA meet the legal requirements to ensure minimum protection against certain contingencies, among which is that of motherhood, and if it empties of content the right of transfer in parent's favour.
\end{abstract}

KEYWORDS: self-employed, maternity leave, Social Security for Independent Workers, Social Protection Mutual.

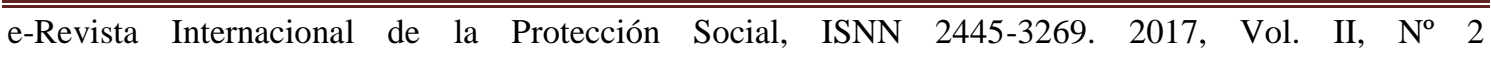




\section{SUMARIO}

I. CRITERIOS DE INTEGRACIÓN EN LA SEGURIDAD SOCIAL DE LOS PROFESIONALES COLEGIADOS.

II. MUTUALIDADES ALTERNATIVAS AL RETA.

A. OPCIÓN DE COBERTURA Y PECULIARIDADES.

B. NIVEL DE COBERTURA Y PRESTACIONES DE LOS MUTUALISTAS: HACIA LA EQUIPARACIÓN CON EL RETA.

III. LA PROTECCIÓN DE LA MATERNIDAD BIOLÓGICA DE LAS TRABAJADORAS AUTÓNOMAS.

A. DIFERENCIAS ENTRE AUTÓNOMAS INCLUIDAS EN EL RETA Y EN UNA MUTUALIDAD ALTERNATIVA.

B. SOBRE LA CESIÓN DEL DERECHO AL DESCANSO AL OTRO PROGENITOR.

C. PLANTEAMIENTO DEL PROBLEMA.

1. CESIÓN DEL DERECHO EN EL SUPUESTO DE TRABAJADORAS AUTÓNOMAS INCLUIDAS EN EL RETA.

2. DERECHO DE CESIÓN EN EL CASO DE PROFESIONALES COLEGIADAS INCLUIDAS EN UNA DE LAS MUTUALIDADES ALTERNATIVAS AL RETA

a) SITUACIÓN PREVIA A LA LEY 27/2011 Y SU POSTERIOR DESARROLLO POR LGSS DE 2015 (DA 19 ${ }^{\mathrm{a}}$ ).

b) LA LIMITADA APLICABILIDAD DE LA MEDIDA AL AMPARO DE LA REGULACIÓN VIGENTE.

D. VISIÓN PANORÁMICA DE LA PRESTACIÓN POR MATERNIDAD DE TRABAJADORAS AUTÓNOMAS Y PROFESIONALES LIBERALES EN OTROS PAÍSES EUROPEOS.

IV. REFLEXIONES FINALES.

\begin{tabular}{llllllllllll}
\hline \hline e-Revista & Internacional de la Protección & Social, & ISNN & $2445-3269$. & 2017, & Vol. & II, & N $^{\circ}$ & 2
\end{tabular} 


\section{CRITERIOS DE INTEGRACIÓN EN LA SEGURIDAD SOCIAL DE LOS PROFESIONALES COLEGIADOS}

Desde la creación del RETA, por Decreto 2530/1970, de 20 agosto (BOE de 15 de septiembre), el proceso de encuadramiento en la Seguridad Social de los profesionales liberales que, para ejercer su actividad, requerían necesariamente su previa colegiación en un Colegio o Asociación ha atravesado tres fases diferenciadas: un periodo inicial de prohibición, que terminó en $1981^{1}$, una segunda fase, de incorporación colectiva y voluntaria, y otra tercera etapa, de encuadramiento individual y obligatorio, aunque con excepciones.

Durante la segunda fase (1981 a 1995), el art. 3 del Decreto 2530/1970, de 20 de agosto, estableció un régimen peculiar de encuadramiento de carácter colectivo y voluntario para todos los profesionales de la misma actividad, por cuanto se exigía que aquella inclusión se realizara "a solicitud de los órganos superiores de representación de dichas entidades y mediante Orden ministerial" ". De no mediar dicha solicitud, la protección social de los interesados quedaba al margen del sistema público de Seguridad Social. El citado régimen constituía una excepción a la regla de inclusión obligatoria en el RETA para el resto de profesionales liberales por cuenta propia que no necesitaran incluirse obligatoriamente en un Colegio o Asociación Profesional, que se llevaba a efecto mediante solicitud individual del propio interesado.

La tercera fase, de inclusión individual y obligatoria, se inicia con la aprobación de la Ley 30/1995, de 30 de noviembre, sobre Ordenación y Supervisión de los Seguros Privados (LOSSP) (BOE de 9 de noviembre), cuya disposición adicional decimoquinta, modificada por Ley 50/1998, declara voluntaria la pertenencia a una Mutualidad de Previsión Social y establece nuevas reglas de incorporación al sistema de los profesionales colegiados, en función de distintas circunstancias (señaladamente, la concreta mutualidad afectada, la fecha de constitución de esta con carácter obligatorio y la de inicio de la actividad profesional por parte del trabajador), lo que daba lugar a una diversidad de situaciones ${ }^{3}$.

A tenor de lo previsto en la citada DA $15^{\mathrm{a}}$ LOSSP, los profesionales con pertenencia obligatoria a un Colegio Profesional cuyo colectivo no haya sido integrado en el RETA debían darse obligatoriamente de alta en la Seguridad Social, de forma similar al resto de trabajadores por cuenta propia. Tan solo se exceptuaba de esta obligación a los colegiados que se hubiesen incorporado a la mutualidad de previsión social establecida por el Colegio profesional, siempre que esta cumpliera los requisitos siguientes ${ }^{4}$ : estar constituida antes del 10 de noviembre de 1995 al amparo del apartado 2 del artículo 1 del Reglamento de Entidades de Previsión Social, aprobado por el Real Decreto

\footnotetext{
${ }^{1}$ El RD 2504/1980, de 24 octubre, modifica el art. 3 del Decreto 2530/1970 poniendo fin a esta prohibición, con efectos de 1 de enero 1981, al tiempo que establece el régimen de incorporación colectiva de todos los integrantes del Colegio profesional en cuestión.

${ }^{2}$ Por esta vía se han incorporado al RETA más de cuarenta profesiones: deportistas de alto nivel, peritos y tasadores de seguros, graduados sociales, ingenieros agrónomos colegiados, ingenieros técnicos, ópticos colegiados, farmacéuticos titulares, veterinarios, vendedores de prensa, religiosos y religiosas de la Iglesia Católica, etc. Luelmo Millán, M.A., "El trabajador autónomo: caracteres generales de su actividad y encuadramiento", Actualidad Laboral, núm. 18, 2002, Ref. XXII, pág. 353, Editorial LA LEY, LA LEY 2025/2002, p. 5.

${ }^{3}$ Lopez Aniorte, M.C., "El encuadramiento de los profesionales colegiados en la Seguridad Social: un proceso inacabado”, Foro de Seguridad Social, núm. 20, 2008, p. 68.
}

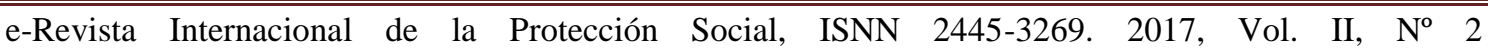


2615/1985, de 4 de diciembre y tener un mecanismo de adscripción obligatoria ${ }^{5}$. Al objeto de dar cumplimiento a dicha obligación de aseguramiento individual los interesados podían optar por solicitar la afiliación y/o el alta en dicho Régimen Especial o incorporarse a la Mutualidad que tenga establecida dicho Colegio Profesional.

Sin embargo, la DA 18 ${ }^{\text {a }}$ LGSS (RD Legislativo 8/2015, 30 octubre; BOE de 31 de octubre)- vigente desde 2 enero 2016- ha supuesto la derogación de la anterior regulación relativa al encuadramiento de los profesionales colegiados. A partir de ahora, quienes ejerzan su actividad por cuenta propia que requiera la incorporación a un colegio profesional cuyo colectivo no hubiera sido integrado en el RETA, se entenderán incluidos en el campo de aplicación del mismo, debiendo solicitar, en su caso, la afiliación y, en todo caso, el alta en dicho régimen especial en los términos establecidos reglamentariamente.

No obstante, la DA $18^{\mathrm{a}}$ LGSS contempla varios supuestos de exención de la obligación de alta en el RETA, en los que se reconoce el derecho de opción entre darse de alta en el RETA o en la Mutualidad de previsión social alternativa.

En cualquiera de los supuestos contemplados en los apartados 1 y 2 de la DA $18^{\text {a }}$ LGSS, la inclusión en el citado régimen especial se llevará a cabo sin necesidad de mediar solicitud previa de los órganos superiores de representación de los respectivos colegios profesionales.

\section{MUTUALIDADES ALTERNATIVAS AL RETA}

\section{A. OPCIÓN DE COBERTURA Y PECULIARIDADES}

La DA $18^{\text {a }}$ LGSS establece, desde el 2 de enero de 2016, la obligatoriedad de la inclusión en el RETA de los profesionales colegiados, aunque se mantiene la exención de la obligación de alta en este Régimen Especial para los colegiados que opten o hubieren optado por incorporarse a la mutualidad de previsión social que pudiera tener establecida el correspondiente colegio profesional, siempre que la citada mutualidad sea alguna de las constituidas con anterioridad al 10 de noviembre de 1995 al amparo del apartado 2 del artículo 1 del Reglamento de Entidades de Previsión Social, aprobado por el Real Decreto 2615/1985, de 4 de diciembre (BOE de 15 de enero de 1986). Si el interesado, teniendo derecho, no optara por incorporarse a la mutualidad correspondiente, no podrá ejercitar dicha opción con posterioridad.

Asimismo, se contempla la exención de encuadramiento obligatorio en el RETA para quienes iniciaran su actividad antes del 10 de noviembre de 1995 y estuvieran integrados en tal fecha en la Mutualidad de Previsión Social que pudiera tener establecida el correspondiente Colegio Profesional, que no se hubiera integrado en el RETA, quienes podrán elegir entre solicitar el alta en dicho régimen especial o permanecer incorporados a la Mutualidad correspondiente en el momento en que se produzca el cambio.

\footnotetext{
${ }^{5}$ La TGSS, mediante Circular de 15 de febrero de 1999, determinó las mutualidades que debían considerarse como alternativas al RETA (a ellas este grupo se incorporó en 2006 el colectivo de médicos) y aquellas otras mutualidades obligatorias en fecha de10 de noviembre de 1995 que no se eran alternativas (Mutualidad del Cuerpo de Corredores de Comercio Colegiados o la del Colegio de Ingenieros de Caminos, Canales y Puertos).

e-Revista Internacional de la Protección $\quad$ Social, ISNN 2445-3269. 2017, Vol. II, $\mathrm{N}^{\circ} \quad 2$ http://dx.doi.org/10.12795/e-RIPS.2017.i02.11.

Página 156
} 
Las Mutualidades que pueden ser una alternativa al RETA, de acuerdo con la legislación vigente son las diez siguientes: a) Alter Mutua ${ }^{6}$; b) Hermandad Nacional de Arquitectos Superiores (HNA), que absorbió a Mutualidad General de Previsión Social de Químicos Españoles; c) Previsión Mutua Aparejadores y Arquitectos Técnicos (PREMAAT); d) Mutualidad de Previsión Social de Procuradores de los Tribunales de España; e) Asociación Mutualista Ingeniería Civil (AMIC); f) Mutual Médica; g) Mutua de Ingenieros Industriales; h) Mutualidad de Gestores Administrativos; i) M.P.S. Peritos Ingenieros Técnicos Industriales (MUPITI); j) Mutualidad de la Abogacía.

En consecuencia, determinados profesionales liberales que ejercen su actividad por cuenta propia como los abogados, médicos, arquitectos, aparejadores, procuradores, gestores administrativos e ingenieros, no están obligados a instar su encuadramiento en la Seguridad Social, ya que, para estos colectivos, existe el derecho de opción entre su incorporación en el RETA o en alguna de las citadas Mutualidades de Previsión Social específicas para su profesión.

La opción que se les plantea es la de poder acogerse exclusivamente a un sistema de capitalización individual de aportación definida o a un sistema de Seguridad Social financiado por reparto o, incluso, la de incorporarse de forma voluntaria en el RETA y mantener su inclusión en tales sistemas privados de previsión social, que tendrían entonces un carácter complementario del sistema público de Seguridad Social.

Dicha posibilidad de opción entre sistema de previsión privado como alternativo al público, y la de mantener ambos de forma complementaria, constituye un privilegio insólito y difícilmente justificable, salvo por razones históricas o por la existencia de una fuerte "presión" procedente de ciertos Colegios profesionales reacios a renunciar a sus prerrogativas ${ }^{7}$.

Entre otras ventajas, las cuotas son más reducidas que las del RETA, aunque se ha establecido una aportación mínima obligatoria, y además, comparativamente, tienen un mejor tratamiento fiscal, ya que las primas o cuotas abonadas a la Mutualidad en su condición de alternativas, en la parte que tenga por objeto la cobertura de las contingencias cubiertas por el mismo, tienen la consideración de gasto deducible de los ingresos de la actividad profesional, con el límite del 100 por 100 de la cuota máxima

\footnotetext{
${ }^{6}$ Orden EHA/1984/2009, de 6 de mayo, autorizando a la entidad Alter Mutua para el ejercicio de la actividad aseguradora como mutua de previsión social en todo el territorio español para la profesión de abogado; y el Oficio de 8 de octubre de 2009, de la Dirección General de Ordenación de la Seguridad Social del Ministerio de Trabajo e Inmigración por el que se reconoce a Alter Mutua la condición de alternativa a la de alta en el RETA en todo el territorio del Estado.

${ }^{7}$ Para algunos autores, más que a razones de puro escapismo del sistema público, la regulación pretende dar continuidad a la función supletoria que ciertas Mutualidades cumplieron en el pasado con respecto a colectivos que, por decisión de sus respectivos colegios profesionales, permanecieron excluidos del sistema e incorporados en aquellas con carácter obligatorio; de ahí la doble exigencia de su "antigüedad" y de su "obligatoriedad" que se establece para su conceptualización como "alternativas". Tejerina Alonso, J.I., "La Seguridad Social de los profesionales colegiados", Revista Actuarios, $\mathrm{n}^{\circ}$ 22, 2004, p. 2 (http://www.actuarios.org/espa/revista22/ss.htm). Por el contrario, para otros, este sistema de opción es inaceptable por discriminatorio y por suponer una ruptura del principio de extensión del ámbito aplicativo del RETA a colectivos completos. En este sentido, López Aniorte, M.C. "Los profesionales colegiados y la Seguridad Social. El lento y complejo camino hacia su completa integración en el sistema", Relaciones Laborales, no 21, 1997, (LA LEY 5971/2002), pág. 11.
}

e-Revista Internacional de la Protección Social, ISNN 2445-3269. 2017, Vol. II, N $^{\circ} 2$ http://dx.doi.org/10.12795/e-RIPS.2017.i02.11.

Página 157 
por contingencias comunes que esté establecida en cada ejercicio económico para el RETA (DA. 19a , apartado 3 LGSS) ${ }^{8}$.

De otro lado, en supuestos de pluriactividad, al estar encuadrados en el sistema por razón del trabajo por cuenta ajena, la permanencia en la Mutualidad les permite evitar duplicar cotizaciones al sistema público, duplicidad de cotizaciones que sin embargo sufren irremediablemente la generalidad de los autónomos que también trabajan por cuenta ajena.

\section{B. NIVEL DE COBERTURA Y PRESTACIONES DE LOS MUTUALISTAS: HACIA LA EQUIPARACIÓN CON EL RETA}

La consideración de determinadas mutualidades de previsión social como alternativas al RETA presentaba el riesgo de que estas no ofrecieran un nivel de protección adecuado dentro del marco estatal, debido a que la LOSSP no establecía ni el tipo ni la cuantía de las prestaciones que deben garantizar a sus afiliados. De hecho, un importante déficit de cobertura se planteaba, por ejemplo, en relación con la contingencia de maternidad.

Sin embargo, en los últimos tiempos esta fórmula de protección social de base mutualista está recibiendo un fuerte respaldo por medio de reformas legales que tratan de mejorar la cobertura que ofrece a los profesionales colegiados para equipararla a la de los cotizantes como autónomos.

Manifestación de esta tendencia es, por ejemplo, la admisión de la capitalización del desempleo para el pago de las cuotas a la mutualidad, tal y como se puede hacer en el caso de autoempleo con las cuotas de autónomos.

Mayor trascendencia tiene sin duda el reconocimiento, mediante RD 1192/2012, de 3 de agosto (BOE de 4 de agosto), por el que se regula la condición de asegurado y beneficiario de la asistencia sanitaria con cargo a fondos públicos en España, del derecho a la sanidad pública a los profesionales acogidos a sus mutualidades de previsión social alternativas en las mismas condiciones que los afiliados, sin necesidad de cotizar a la Seguridad Social, mediante la suscripción de un convenio especial (aunque limitado a aquellos mutualistas con ingresos inferiores a los 100 mil euros anuales). Es obvio que, con esta equiparación, se elimina uno de los inconvenientes que tenía la opción por la Mutualidad del colegio profesional. Ahora bien, al desaparecer la necesidad de darse de alta en el RETA o de suscribir un convenio especial para poder tener acceso a la sanidad pública, se están restando recursos económicos al sistema de Seguridad Social procedentes de las cuotas de los profesionales colegiados.

Por último, en desarrollo del mandato contenido en la Disposición Adicional $46^{\mathrm{a}}$ de la Ley 27/2011, de 1 de agosto (BOE de 2 de agosto), se han fijado, a partir de 2013, unos niveles mínimos de cobertura de contingencias cubiertas y prestaciones que deben necesariamente ofrecer las mutualidades para poder ser consideradas alternativas al alta en el RETA, los cuales han sido actualizados mediante la Disposición Adicional $19^{\mathrm{a}}$ de la vigente LGSS.

Así, de un lado, en lo que se refiere a las contingencias cubiertas, el apartado 1 de la DA $19^{a}$ LGSS, establece que "dichas mutualidades deberán ofrecer a sus afiliados, mediante

\footnotetext{
${ }^{8}$ En 2016, el límite deducible es de 13.067,52 euros. Además, si el mutualista desea hacer una aportación mayor, este exceso se podrá deducir de la base imponible general del IRPF. Jiménez González, J., "Previsión Social y seguros personales: Mutualidades alternativas a Autónomos. Luces y sombras", (http://mutualidades.blogspot.com.es/2015/03/mutualidades-alternativas-autónomos.html; consultado el 08/02/2016), pág. 2.

e-Revista Internacional de la Protección Social, ISNN 2445-3269. 2017, Vol. II, $\mathrm{N}^{\circ} \quad 2$ http://dx.doi.org/10.12795/e-RIPS.2017.i02.11.

Página 158
} 
el sistema de capitalización individual y la técnica aseguradora bajo los que operan, de forma obligatoria, las coberturas de jubilación; incapacidad permanente; incapacidad temporal, incluyendo maternidad, paternidad y riesgo durante el embarazo; $y$ fallecimiento que pueda dar lugar a viudedad y orfandad".

Si comparamos el ámbito obligatorio de cobertura exigido a las Mutualidades de previsión social alternativas con el definido en el art. 314 LGSS para los trabajadores por cuenta propia incluidos en el $\operatorname{RETA}^{9}$, se observa que únicamente se dejan fuera del ámbito de protección de dichas mutualidades las contingencias de riesgo durante la lactancia natural, por cese de la actividad y la de cuidado de menores con cáncer u otra enfermedad grave.

De otro lado, en relación con el nivel de protección, se establecen también unas coberturas mínimas que deben necesariamente ofrecer las mutualidades alternativas al RETA. Tales niveles mínimos de cobertura se fijan en función de dos criterios alternativos: bien sea el importe de la prestación a satisfacer por la citada mutualidad, o bien, el importe de la cuota a abonar por el mutualista.

Así, en primer lugar, el apartado 2 de la DA 19ª LGSS, en su primer párrafo, establece que "las prestaciones que se otorguen por las mutualidades en su condición de alternativas al citado régimen especial, cuando adopten la forma de renta, habrán de alcanzar en el momento de producirse cualquiera de las contingencias cubiertas a que se refiere el apartado anterior, un importe no inferior al 60 por ciento de la cuantía mínima inicial que para la respectiva clase de pensión rija en el sistema de la Seguridad Social o, si resultara superior, el importe establecido para las pensiones no contributivas de la Seguridad Social. Si tales prestaciones adoptaran la forma de capital, este no podrá ser inferior al importe capitalizado de la cuantía mínima establecida para caso de renta”.

En relación a este primer criterio, cabe hacer varias observaciones:

$1^{\mathrm{a}}$. Dicho criterio únicamente sirve para conocer si la cuantía de las pensiones que ofrecen las Mutualidades (jubilación, incapacidad permanente, viudedad y orfandad) es o no adecuada, puesto que son las pensiones las que tienen garantizadas cuantías mínimas en el Sistema de la Seguridad Social.

$2^{\text {a }}$. La cuantía mínima de las pensiones que oferte la Mutualidad correspondiente no podrá ser inferior al 60 por ciento de la cuantía mínima inicial que para la respectiva clase de pensión rija en el sistema de la Seguridad Social. Ello significa que habrá que atender a la concreta clase de pensión - de jubilación, incapacidad permanente contributiva, viudedad u orfandad- y también a los demás elementos que, en su caso, inciden en la cuantía mínima de la pensión de

\footnotetext{
${ }^{9} \mathrm{El}$ art. 42 LGSS dispone que la acción protectora del sistema de Seguridad Social comprenderá: "Las prestaciones económicas en las situaciones de incapacidad temporal; maternidad; paternidad; riesgo durante el embarazo; riesgo durante la lactancia natural; cuidado de menores afectados por cáncer u otra enfermedad grave; incapacidad permanente contributiva e invalidez no contributiva; jubilación, en sus modalidades contributiva y no contributiva; desempleo, en sus niveles contributivo y asistencial; protección por cese de actividad; muerte y supervivencia; así como las que se otorguen en las contingencias y situaciones especiales que reglamentariamente se determinen por real decreto, a propuesta del titular del Ministerio de Empleo y Seguridad Social”. Por su parte, el art. 314 LGSS establece que la acción protectora en el RETA "será la definida en el art. 42 LGSS, con excepción de la protección por desempleo y las prestaciones no contributivas".
}

e-Revista Internacional de la Protección Social, ISNN 2445-3269. 2017, Vol. II, N $^{\circ} \quad 2$ http://dx.doi.org/10.12795/e-RIPS.2017.i02.11.

Página 159 
que se trate (edad del pensionista, si tiene o no cónyuge a cargo o es una unidad unipersonal, grado de discapacidad, la existencia de hijos a cargo, etc.) ${ }^{10}$.

$3^{\text {a }}$. El requisito de que la cuantía de la pensión ofertada por la Mutua del Colegio profesional sea equivalente, como mínimo, al 60 por ciento de la correspondiente pensión mínima del sistema de la Seguridad Social no se aplicará si resulta superior al importe de la pensión no contributiva ${ }^{11}$. Este segundo límite corrector se aplicará en relación con la mayoría de las pensiones, puesto que el 60 por ciento de las correspondientes cuantías mínimas resulta superior a la pensión no contributiva. Así, sucede con la pensión de jubilación para beneficiarios con 65 años (salvo en el supuesto de que tenga cónyuge no a cargo), y para beneficiarios menores de 65 años (aunque únicamente cuando tenga cónyuge a cargo); también en caso de pensionistas de gran invalidez, para incapacidad permanente absoluta y total cuyos beneficiarios tengan 65 años o entre 60 y 64 años (salvo en el supuesto de que tenga cónyuge no a cargo o cuando se trate de pensión de incapacidad permanente total derivada de enfermedad común siendo menores de 60 años); finalmente, tampoco se aplicaría para la pensión de viudedad (salvo para beneficiarios menores de 65 años sin cargas familiares ni discapacidad en grado igual o superior al 65 por ciento).

4a.- La cuantía mínima establecida para el caso de renta sirve también para determinar el nivel mínimo de las prestaciones que adopten la forma de capital, que no podrá ser inferior al importe capitalizado de la referida cuantía mínima.

$5^{\text {a }}$ - El criterio examinado permite un amplio margen para la cobertura de las contingencias cubiertas, por lo que puede considerarse vigente la doctrina sentada por el Tribunal Supremo (STS 16 mayo 20101; Recurso núm. 1455/2000; TJ 2001, 5216), conforme a la cual las Mutualidades no están obligadas a ofrecer el mismo nivel de protección que el sistema público de Seguridad Social, ni por tanto, no cabe exigirles que garanticen la cuantía mínima de las pensiones que el Estado garantiza cada año a las pensiones públicas $^{12}$.

$6^{\text {a }}$ - El criterio no permite determinar el nivel mínimo de cobertura de las prestaciones de carácter temporal, como incapacidad temporal, maternidad, paternidad y riesgo durante el embarazo, al no tener estas señaladas cuantías mínimas. Aunque el legislador podía haber fijado un nivel mínimo de cobertura de estas prestaciones, situándolo por ejemplo, en un concreto porcentaje de la base mínima de cotización, lo cierto es que, no se sabe si por olvido o conscientemente, no es esta la solución prevista. En consecuencia, la valoración

\footnotetext{
${ }^{10}$ Las cuantías de las pensiones mínimas para 2016 se regulan en el Anexo del RD 1170/2015, 29 diciembre, sobre revalorización de las pensiones del sistema de la seguridad Social. A título de ejemplo, si se trata de una pensión de orfandad, dado que la cuantía mínima por beneficiario en 2016 es igual a 194,30 euros/mensuales, respecto de la pensión de orfandad que oferte una Mutualidad de Previsión Social alternativa al RETA, se considerará que su importe mínimo es adecuado si es igual al 60 por ciento de dicha cantidad, esto es, 116,58 euros mensuales.

${ }^{11}$ Por lo tanto, en 2016, la cuantía mínima de las pensiones de la mutualidad no podrá ser superior a 367,90 euros mensuales (5.150,60 euros anuales).

${ }^{12}$ Ferrando García, F.M., "Las Mutualidades de Previsión Social: complemento y alternativa al sistema público de Seguridad Social”, en AAVV. (Ma . Mar Andreu Martí, Dir. y Coord.), La empresa social y su organización jurídica, Marcial Pons, Madrid, 2014, p. 274.
}

e-Revista Internacional de la Protección Social, ISNN 2445-3269. 2017, Vol. II, $\mathrm{N}^{\circ} 2$ http://dx.doi.org/10.12795/e-RIPS.2017.i02.11.

Página 160 
del nivel de cobertura de estas prestaciones deberá realizarse conforme al segundo criterio.

La misma Disposición adicional 19 ${ }^{\mathrm{a}}$ LGSS (apartado 2, segundo párrafo), prevé un segundo criterio para determinar la cobertura mínima obligatoria, que vincula el importe de las cuotas a ingresar por los mutualistas a las que se abonan en el sistema público, y lo hace en los siguientes términos: "se considerará, asimismo, que se cumple con la obligación de cuantía mínima de la prestación, si las cuotas a satisfacer por el mutualista, cualesquiera que sean las contingencias contratadas con la mutualidad alternativa, de entre las obligatorias a que se refiere el apartado 1, equivalen al 80 por ciento de la cuota mínima que haya de satisfacerse con carácter general en este régimen especial"13.

Esta exigencia produce un efecto doble. De un lado, se evita que se abonen unas cuotas irrisorias que impidan a las Mutualidades ofrecer el nivel de protección que se estima adecuado para que pueda considerarse alternativas al sistema público de Seguridad Social, lo que supone una mejora de la protección para los mutualistas. Pero, de otro lado, con la subida de las cuotas a satisfacer a las Mutualidades y acercarlas a la del RETA, parece que se está forzando a los profesionales colegiados a optar por darse de alta en este Régimen Especial, y quedar así encuadrados en un sistema público de Seguridad Social, con un ámbito objetivo de cobertura más extenso, de mayor intensidad protectora y mayores garantías de pervivencia futura.

\section{LA PROTECCIÓN DE LA MATERNIDAD BIOLÓGICA DE LAS TRABAJADORAS AUTÓNOMAS}

\section{A. DIFERENCIAS ENTRE AUTÓNOMAS INCLUIDAS EN EL RETA Y EN UNA MUTUALIDAD ALTERNATIVA}

El régimen jurídico de las prestaciones por maternidad biológica de las trabajadoras autónomas difiere según estén integradas en el RETA o en la Mutualidad del Colegio Profesional.

En efecto, mientras que respecto de las primeras, existe una equiparación casi plena con las trabajadoras por cuenta ajena, en cambio, en el caso de las profesionales colegiadas no incluidas en el RETA, el derecho a la prestación por maternidad se reconoce- desde 2013- en las condiciones que establezca la mutualidad y con diverso alcance y contenido que en el sistema de la Seguridad Social.

En relación con los trabajadores autónomos encuadrados en el RETA, el art. 318 del vigente TRLGSS, declara aplicables a este régimen especial, en materia de maternidad y paternidad lo dispuesto en los capítulos VI y VII del título II, respectivamente (que regulan las prestaciones de maternidad y paternidad en el Régimen General de la Seguridad Social). Este mismo precepto, señala que "Los períodos durante los que el trabajador por cuenta propia tendrá derecho a percibir los subsidios por maternidad y paternidad serán coincidentes, en lo relativo tanto a su duración como a su distribución, con los períodos de descanso laboral establecidos para los trabajadores por cuenta ajena, pudiendo dar comienzo el abono del subsidio por paternidad a partir del momento del

\footnotetext{
${ }^{13}$ Según la Orden de cotización para 2016 (Orden ESS 70/2016, 29 enero), este la base mínima de cotización de los autónomos, que es por la que cotiza el 86\% del colectivo, se sitúa en 2016 en 893,10 euros y el tipo a aplicar, incluida la incapacidad temporal y financiación de prestaciones por riesgo durante el embarazo y la lactancia, sería el 29,90\% de 893,10 euros, lo que conlleva, una cuota mínima mensual de 267,04 euros con carácter general. Por tanto, el 80 por ciento de dicha cuota equivale a 213,63 euros mensuales.

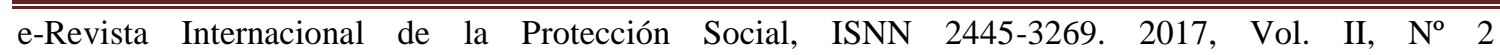
http://dx.doi.org/10.12795/e-RIPS.2017.i02.11.

Página 161
} 
nacimiento del hijo. Los trabajadores de este régimen especial podrán igualmente percibir los subsidios por maternidad y paternidad en régimen de jornada parcial, en los términos y condiciones que se establezcan reglamentariamente".

Por lo tanto, la protección por maternidad en el RETA es idéntica a la prevista en el RGSS en lo referente a situaciones protegidas, carencia exigida, cuantía y duración. Tan solo existen tres peculiaridades relacionadas con la naturaleza autónoma de la actividad desarrollada. Dos ellas se refieren a la exigencia de dos requisitos adicionales de acceso a las prestaciones (la necesidad de estar al corriente en el pago de las cuotas y la de presentación de declaración de situación de actividad) y, la tercera, está relacionada con el disfrute del descanso en régimen de jornada a tiempo parcial, regulado en el apartado 8 de la Disposición Adicional Primera del RD 295/2009, de 6 de marzo (BOE de 21 de marzo) $)^{14}$.

La situación es muy distinta en lo que concierne a las profesionales colegiadas incluidas en una Mutualidad alternativa, pues, hasta enero de 2013, aquellas no protegían esta contingencia con carácter obligatorio. La única posibilidad que tenían tales mutualistas consistía en contratar un seguro privado, que en cualquier caso, exigía un elevado período de carencia previa ${ }^{15}$.

Sin embargo, a partir de 2013, para mantener su carácter de alternativas al RETA, las citadas Mutualidades de Previsión Social ha tenido que incorporar obligatoriamente la contingencia de maternidad y garantizar un nivel mínimo de cobertura.

\section{B. SOBRE LA CESIÓN DEL DERECHO AL DESCANSO AL OTRO PROGENITOR.}

\section{PLANTEAMIENTO DEL PROBLEMA}

En supuestos de parto, nuestra legislación sigue configurando a la madre trabajadora por cuenta ajena como titular del derecho a la suspensión del contrato por maternidad.

Con el fin de favorecer la igualdad de trato en el trabajo, la Ley 3/1989, de 3 marzo (BOE de 8 de marzo), introdujo la posibilidad de disfrute paterno del descanso por maternidad biológica por fallecimiento de la madre o por cesión de esta, y, la LO $3 / 2007$, de 22 de marzo (BOE de 23 de marzo), para la igualdad efectiva de mujeres y hombres (art. 44), añadió también el supuesto de cesión por ausencia de derecho de la madre (nuevo párrafo adicionado al art. 48.4 ET). Desde entonces, la cuestión del disfrute de dicho permiso por el otro progenitor ha suscitado una no desdeñable litigiosidad $^{16}$.

\footnotetext{
${ }^{14}$ López Aniorte, M.C. Y Rodríguez Egío, M.M., “¿Favorece el emprendimiento femenino el vigente régimen jurídico de la protección por maternidad biológica?", Revista Derecho Social, núm. 70 2015, pp. 85-92.

${ }^{15}$ Así, por ejemplo, en la Mutualidad de la Abogacía, a partir de 2005 se contempló la protección por maternidad con el requisito de un periodo previo de carencia de suscripción voluntaria de un seguro privado, que garantizaba el pago de una indemnización cuando la profesional de la abogacía se viera obligada a interrumpir totalmente su actividad por razón de maternidad. Este sistema voluntario se mantuvo hasta la entrada en vigor de la Ley 27/2011 (DA 46a).

${ }^{16}$ Lousada Arochena, J.F., "El reglamento de las prestaciones por maternidad y paternidad: puntos críticos", Aequalitas: Revista jurídica de igualdad de oportunidades entre hombres y mujeres, $\mathrm{N}^{\circ} 24$, 2009 , p. 51.

e-Revista Internacional de la Protección Social, ISNN 2445-3269. 2017, Vol. II, $\mathrm{N}^{\circ} 2$ http://dx.doi.org/10.12795/e-RIPS.2017.i02.11.

Página 162
} 
Con relación a este tema, la jurisprudencia ha hecho tradicionalmente una interpretación restrictiva de la norma al exigir que ambos progenitores trabajen, teniendo que reunir cada uno de ellos los requisitos de afiliación, alta y carencia como trabajadores con vinculación de naturaleza laboral y que estén incluidos en el Régimen General de la Seguridad Social (u otro que incluya trabajadores por cuenta ajena), o funcionarios o asimilados.

Ahora bien, con respecto a las trabajadoras autónomas encuadradas en el RETA, la referida doctrina debe considerarse superada a tenor de lo dispuesto en el art. 1 del RD 295/2009, de 6 de marzo, que extiende la normativa sobre la opción a favor del otro progenitor a todos los regímenes del sistema ${ }^{17}$. En cambio, habría que considerar que no pueden transmitir el derecho del que carecen al otro progenitor las profesionales liberales incorporadas a la Mutualidad del Colegio Profesional, al estar excluidas del sistema de la Seguridad Social.

\section{DERECHO DE CESIÓN EN EL SUPUESTO DE TRABAJADORAS AUTÓNOMAS INCLUIDAS EN EL RETA}

A pesar de que con anterioridad a la entrada en vigor de la Ley de Igualdad, el fundamento del derecho de cesión de la autónoma integrada en el sistema al otro progenitor podría encontrarse en la Disposición Adicional Undécima bis de la LGSS de 1994 (RD Legislativo 1/1994, de 20 de junio; BOE de 29 de junio) ${ }^{18}$, sorprendentemente, el Tribunal Supremo ha venido considerando que únicamente las trabajadoras por cuenta ajena vinculadas mediante contrato de trabajo tienen reconocido el derecho a ceder al otro progenitor parte del descanso y del correspondiente subsidio, denegando a las trabajadoras por cuenta propia la posibilidad de hacerlo ${ }^{19}$.

Siguiendo la doctrina de unificación de la Sala IV del Tribunal Supremo, así lo entiende, también, el TSJ del País Vasco (Social) en sus Sentencias de 20 de febrero de 2001 (AS 2001, 4346), y 27 de marzo de 2001 (JUR 2001, 300567) en relación a dos trabajadoras no incluidas en el sistema de la Seguridad Social, estando la segunda de dichas Sentencias referida a abogada afiliada a la Mutualidad General de la Abogacía. Idéntica postura denegatoria del derecho del padre -trabajador por cuenta ajena- a la prestación por maternidad en supuestos de madre trabajadora por cuenta propia afiliada al RETA mantienen, entre otras, las STSJ del País Vasco, de 16 de abril de 2002 (JUR 2002, 221391) y también STSJ Andalucía/Granada, de 18 diciembre 2000 (AS 2001, 970), STSJ Murcia, de 30 septiembre 2002 (AS 2002, 3004), y STSJ Madrid, de 26 febrero 2003 (AS 2002, 3015) $)^{20}$.

Sin embargo, con posterioridad a la entrada en vigor de la Ley de igualdad, en 2007, esta sólida doctrina judicial contraria al reconocimiento del derecho de cesión a la autónoma integrada en el sistema empieza a debilitarse a medida que surge una nueva línea interpretativa de los tribunales de suplicación (sin que el TS haya dictado una

\footnotetext{
${ }^{17}$ Dicho precepto establece:"las disposiciones establecidas en los capítulos II, III, IV y V de este Real Decreto serán de aplicación a todos los regímenes del sistema de la Seguridad Social, sin más particularidades que las que expresamente se indican".

${ }^{18}$ López Aniorte, M.C. Y Rodríguez Egío, M.M., “¿Favorece el emprendimiento femenino el vigente régimen jurídico de la protección por maternidad biológica?", est, cit., p. 96.

${ }^{19}$ SSTS 20 noviembre 2001 (RJ 2002/360) y 28 diciembre de 2000 (RJ 2001/1882), recaídas ambas en RCUD.

${ }^{20}$ Nuñez Cortés-Contreras, P. y Cebrián Carrillo, A., El reflejo en la jurisprudencia de las medidas de conciliación de vida laboral y personal, Consejería de Empleo y Mujer, Comunidad de Madrid, Madrid, 2006 , p. 35.

e-Revista Internacional de la Protección Social, ISNN 2445-3269. 2017, $\begin{array}{lllll}\text { Vol. } & \text { II, } & \mathrm{N}^{\circ} & 2\end{array}$ http://dx.doi.org/10.12795/e-RIPS.2017.i02.11.

Página 163
} 
doctrina unificada)- encabezada por la STSJ País Vasco de 2 de diciembre de 2008 (JUR 2009/305865)- que admite ese derecho de cesión, por entender que las trabajadoras por el hecho de serlo en cuanto encuadradas en los distintos regímenes de la Seguridad Social, tienen reconocido el derecho a la prestación de maternidad con igual extensión, y poseen iguales motivos para optar a que sea el otro progenitor el que disfrute el permiso"

Esta nueva corriente interpretativa, unida a lo dispuesto en el art. 318 de la vigente LGSS y a la previsión del art. 1 del RD 295/2009- en la que se extiende el derecho de opción al otro progenitor a todos los regímenes del sistema-, permite considerar resuelto el dilema y afirmar que las trabajadoras autónomas tienen reconocido este derecho de conciliación de la vida laboral y familiar en igualdad de condiciones que las mujeres por cuenta ajena ${ }^{22}$.

\section{DERECHO DE CESIÓN EN EL CASO DE PROFESIONALES COLEGIADAS INCLUIDAS EN UNA DE LAS MUTUALIDADES ALTERNATIVAS AL RETA \\ a) SITUACIÓN PREVIA A LA LEY 27/2011 Y SU POSTERIOR DESARROLLO POR LGSS DE 2015 (DA 19 $)$}

Hasta la aprobación de la Ley 27/2011 (DA 46 ${ }^{\mathrm{a}}$ ) y su reciente concreción por la DA 19 ${ }^{\mathrm{a}}$ del nuevo TRLGSS, las Mutualidades de los colegios profesionales, pese a su naturaleza de alternativas al RETA, presentaban un grave déficit de cobertura en lo que se refiere específicamente a la contingencia de maternidad, ya que no reconocían ninguna prestación económica que permitiera compatibilizar el ejercicio de la profesión durante los primeros meses posteriores al parto. Esta carencia se ha ido haciendo cada vez más visible a medida que se ha ido incrementando en los últimos años la colegiación de mujeres jóvenes y, por tanto en edad fértil.

Ante la ausencia de derecho de la trabajadora autónoma colegiada, el RD 295/2009 reconoce, en su art. 3.4, un derecho directo al otro progenitor, quien podrá percibir el subsidio contributivo por maternidad que hubiera correspondido a la madre, si reúne todos los requisitos exigidos y disfruta del correspondiente período de descanso, que será, además, compatible con el disfrute de la prestación por paternidad.

\section{b) LA LIMITADA APLICABILIDAD DE LA MEDIDA AL AMPARO DE LA REGULACIÓN VIGENTE}

De acuerdo con el art. 3.4 del RD 295/2009, en el caso de las profesionales colegiadas incorporadas a la Mutualidad como alternativa al RETA, la cesión al otro progenitor únicamente podrá tener lugar cuando aquella "no tuviera derecho a prestaciones por no estar prevista la protección por maternidad en la correspondiente mutualidad", situación a la que se equiparan los supuestos en que "la interesada, por causas ajenas a su voluntad, no reuniera las condiciones exigidas para la concesión de la prestación a cargo de la mutualidad, pese a haber optado por incluir la protección por maternidad desde el momento en que pudo ejercitar dicha opción, con ocasión del ejercicio de la actividad profesional".

\footnotetext{
${ }^{21}$ Sentencia comentada por Olarte Encabo, S., "Transferencia de la prestación por maternidad de las trabajadoras autónomas: la equiparación plena con las trabajadoras por cuenta ajena", Revista Doctrinal Aranzadi Social, núm. 57, 2000.

${ }^{22}$ López Aniorte, M.C. y Rodríguez Egío, M.M., “¿Favorece el emprendimiento femenino...”, est. cit., p. 97.

e-Revista Internacional de la Protección Social, ISNN 2445-3269. 2017, Vol. $\quad$ II, $\quad \mathrm{N}^{\circ} \quad 2$ http://dx.doi.org/10.12795/e-RIPS.2017.i02.11.

Página 164
} 
Sin embargo, la norma reglamentaria niega al otro progenitor el derecho a lucrar el subsidio en el sistema de la Seguridad Social en caso de que "la madre tuviese derecho a prestaciones por maternidad en el sistema de previsión derivado de su actividad profesional, independientemente de su duración o de su cuantía, o cuando no alcanzara este derecho por no haber incluido voluntariamente la cobertura de esta prestación".

En consecuencia, tras la fijación de un nivel mínimo de cobertura y prestaciones por la Ley 27/2011 (DA 46 ), concretado en la DA 19 LGSS, surge la duda de la aplicabilidad del derecho del padre al disfrute del período de descanso por maternidad ex art. 48.4 ET y art. 3.4 RD 295/2009, en el supuesto de profesionales colegiadas, toda vez que, por mandato legal, las Mutualidades de los Colegios reconocen, desde el 1 de enero de 2013, el derecho a la prestación por maternidad.

La respuesta debe ser negativa cuando la madre tiene derecho a la protección por maternidad en el ámbito de cobertura de su colegio profesional, siendo irrelevante su cuantía y duración y/o la forma de la prestación y de que esta dicha prestación se incardine dentro de la "incapacidad temporal profesional", pues ello no le hace perder su autonomía en el ámbito de la acción protectora [STSJ País Vasco (Social), de 17 julio 2014 (REC 1259/2001), STSJ de Asturias (Social), de 13 de junio de 2014 (LA LEY 80231/2014) ${ }^{23}$, y STSJ Castilla León/Burgos (Social), de 8 abril 2015 (LA LEY 38776/2015)].

Todas las mutualidades alternativas se han adaptado a las exigencias de nivel mínimo de cobertura obligatoria, tanto de carácter cualitativo (contingencias cubiertas) como de carácter cuantitativo (cuantía mínima de las pensiones o de las cuotas). Ahora bien, cada una de dichas entidades de seguro establecen distintos requisitos de acceso, cuantía y duración de la prestación, aunque, en general, esta consiste en un pago único. Así, a título de ejemplo, desde enero de 2013, la Mutualidad de la Abogacía reconoce una prestación de maternidad o adopción consistente en un pago único, equivalente a 60 días del capital suscrito. Con carácter general, la prestación por maternidad será de 1.800 euros (equivalente a 30 días), que se percibe además sin necesidad de cursar baja por descanso. En el caso de la Mutualidad de los Ingenieros (AMIC), se reconoce el derecho a la prestación por maternidad consistente en un pago único por hijo equivalente a 400 euros. Por su parte, la Mutualidad para profesionales de la arquitectura técnica (PREMAAT) reconoce el derecho a un pago único en concepto de maternidad biológica de 1.000 euros.

Para los tribunales, aunque la prestación no consista, como en el caso del RETA o del RGSS en un subsidio del 100 por 100 de la base reguladora a abonar durante 16 semanas de descanso, sino en una indemnización a tanto alzado por parto, puede considerarse cubierta dicha contingencia en la medida que cumpla con las garantías establecidas actualmente en la DA 19 LGSS $^{24}$. En este caso, el nivel mínimo debe

\footnotetext{
${ }^{23}$ Así, la STSJ de Asturias (Social), de 13 de junio de 2014 (LA LEY 80231/2014), considera que no se da el presupuesto de cesión en el supuesto de abogada incluida en la Mutualidad General de la Abogacía, por cuanto esta otorgaba en concepto de maternidad por parto -previo al cumplimiento de un periodo de carencia de 300 días (diez meses) desde la fecha de efectos hasta el inicio del periodo de gestación- una cantidad a tanto alzado de 450 euros con independencia de que la madre se halle o no abocada a guardar descanso. Además, en el momento del hecho causante del caso enjuiciado no se encontraban vigentes las garantías de cobertura mínima establecidas en la DA 46ª Ley 27/2011.

${ }^{24}$ La STS Aragón (Social), de 29 abril 2015 - Sentencia número 263/2015 (LA LEY 52478/2015) declara que la función que cumple la prestación dispensada por la Mutualidad es la de sustituir las rentas dejadas de percibir por la madre durante los sesenta días siguientes al parto, aunque se abone en un único pago, siendo la misma que cumple el subsidio de maternidad del sistema de la Seguridad Social, aun con

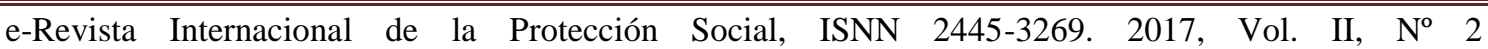
http://dx.doi.org/10.12795/e-RIPS.2017.i02.11.

Página 165
} 
considerarse garantizado, con independencia de la cuantía de la prestación, si las cuotas a abonar a la respectiva Mutualidad son del 80 por ciento de la cuota mínima a abonar en el RETA.

Por lo tanto, únicamente será aplicable cuando "la interesada, por causas ajenas a su voluntad, no reuniera las condiciones exigidas para la concesión de la prestación a cargo de la mutualidad, pese a haber optado por incluir la protección por maternidad desde el momento en que pudo ejercitar dicha opción, con ocasión del ejercicio de la actividad profesional". Así sucederá cuando no reúna la carencia establecida en la correspondiente Mutualidad para lucrar dichas prestaciones ${ }^{25}$.

\section{VISIÓN PANORÁMICA DE LA PRESTACIÓN POR MATERNIDAD DE TRABAJADORAS AUTÓNOMAS Y PROFESIONALES LIBERALES EN OTROS PAÍSES EUROPEOS}

A título meramente ilustrativo se ofrecen unas pinceladas del modo en que queda configurado el derecho a la prestación por maternidad de las profesionales liberales en tres países de la Unión Europea como es el caso de Francia, Italia y Bélgica.

En Francia, las mujeres profesionales liberales están incluidas dentro del régimen de la maternidad de profesionales autónomos, debiendo cotizar al Régimen Social de los Independientes (RSI). En virtud del decreto $n^{\circ}$ 2008-1410, 19 de diciembre de 2008, aquellas tienen derecho a una ampliación de la duración mínima del descanso por maternidad establecida en cuarenta y cuatro días consecutivos, de los cuales, 14 días debe tomarse antes de parto. Este período podrá ser prorrogado a solicitud del interesado por uno o dos períodos de quince días consecutivos ${ }^{26}$.

Sin embargo, las profesionales liberales, que pertenecen al colectivo de practicantes y asistentes médicos, vinculadas mediante convenio con la Seguridad Social, tienen derecho a partir del Decreto 2006-644, del 1 de junio de 2006, a un periodo de descanso por maternidad igual al de trabajadores por cuenta ajena. Tales trabajadoras percibirán prestaciones a tanto alzado de descanso maternal destinadas a compensar parcialmente la disminución de la actividad, que se abonan en dos pagos por un importe equivalente al tope máximo establecido por la seguridad Social en caso de nacimiento y a la mitad en caso de adopción. En el supuesto de interrupción total de la actividad, tienen derecho

una duración superior y un modo de pago diferente. Por ello niega al padre el derecho al disfrute del subsidio por maternidad del sistema Público.

${ }^{25}$ En la Mutualidad de la Abogacía, en el supuesto de maternidad el periodo de carencia dependerá del sistema en el que está contratada la cobertura. En el Sistema de Previsión Profesional no se considerarán períodos de carencia cuando el alta a efectos de la cobertura de la contingencia en el sistema como alternativo coincida con el alta en el sistema. En el resto de sistemas, el período de carencia es de 24 meses desde la contratación hasta el inicio del período de gestación o de la fecha de la resolución por la que se constituye la adopción. Igualmente, para la cobertura de la maternidad, adopción legal y/o enfermedades derivadas o agravadas por embarazo, aborto o parto, el Sistema de Previsión Personalizado (SPP) de los arquitectos por cuenta propia exige un periodo de carencia de 24 meses. Sin embargo, hay pronunciamientos en sentido contrario que estiman que el pago único no tienen la naturaleza de prestación sustitutoria [STS Asturias $n^{\circ}$ 1284/2014, de 13 de junio (La LEY 80231/2014); STSJ Andalucía/Sevilla no 1875/2014, de 2 de julio (LA LEY 123874/2014), entre otras]. Asimismo, la Resolución de los Servicios Centrales del INSS de 16 de junio de 2009 rechazó atribuir la naturaleza de subsidio por maternidad al exiguo pago de 150,25 euros que, por dicho concepto, abonaba la Mutua de Aparejadores y Arquitectos Técnicos.

${ }^{26} \mathrm{http}: / /$ droit-finances.commentcamarche.net/faq/5804-professions-liberales-et-conge-maternite http://www.cdomk21.org/cms/images/stories/pdf_cdo21/CONGE_LIBERAL_DE.pdf.

e-Revista Internacional de la Protección Social, ISNN 2445-3269. 2017, Vol. II, $\mathrm{N}^{\circ} \quad 2$ http://dx.doi.org/10.12795/e-RIPS.2017.i02.11.

Página 166 
a una prestación diaria equivalente a 1/60 parte del tope máximo establecido por la Seguridad Social.

De otro lado, en Italia, la protección por maternidad de las trabajadoras autónomas y profesionales liberales, se regula, respectivamente en los capítulos XI (arts. 66-69) y XII (arts. 70 y ss.) del Decreto legislativo de 26 de marzo de 2001, núm. 151 ${ }^{27}$. De acuerdo con lo previsto en el art. 70, a los profesionales liberales encuadrados en el sistema de Seguridad Social a través del Instituto Nacional de Previsión Social (INPS), se les abonará un subsidio de maternidad durante dos meses antes del nacimiento y tres meses posteriores al mismo. La cuantía de la prestación equivale a un porcentaje de los ingresos profesionales declarados a efectos fiscales como ingresos en el segundo año anterior al hecho causante, si bien dicho importe está sujeto a unos límites mínimos y máximos.

Por último, en Bélgica, la duración máxima del descanso por maternidad para los trabajadores por cuenta propia es de ocho semanas -frente a las quince reconocidas a los trabajadores por cuenta ajena-, de las cuales tres deben disfrutarse obligatoriamente antes del parto y dos semanas posteriores al parto (los asalariados pueden disfrutar seis semanas previas al parto, de las cuales cinco son facultativas y pueden ser trasladadas al periodo posterior al parto, pero una es de descanso obligatorio anterior al parto). En cuanto a la cuantía, a diferencia de los trabajadores por cuenta ajena para quienes el importe de la prestación es igual a un porcentaje del salario bruto (del $82 \%$ durante los primeros 30 días y del 75\% a partir del día 31, con un máximo de 98,70 euros/día), para los autónomos, la cuantía es igual a un pago único equivalente a 440,50 euros por cada semana de descanso ${ }^{28}$.

\section{REFLEXIONES FINALES}

El encuadramiento de los profesionales colegiados se regula, desde enero de 2016, en la Disposición adicional $18^{\text {a }}$ del Texto refundido de la LGSS (aprobado por RD Leg. $8 / 2015$, de 30 de octubre). Como la anterior regulación, tras declarar la inclusión individual y obligatoria de estos en el RETA, admite, a continuación, varias exenciones. En consecuencia, la normativa vigente sigue reconociendo la posibilidad de opción entre sistema de previsión privado como alternativo al público. Ello constituye un privilegio insólito y difícilmente justificable, por cuanto contradice el principio de encuadramiento obligatorio en el sistema de Seguridad Social.

La consideración de determinadas mutualidades de previsión social como alternativas al RETA presentaba el riesgo de que estas no ofrecieran un nivel de protección adecuado dentro del marco estatal. De hecho, un importante déficit de cobertura se planteaba, por ejemplo, en relación con la contingencia de maternidad. Sin embargo, en los últimos tiempos esta fórmula de protección social de base mutualista está recibiendo un fuerte respaldo mediante reformas que han mejorado la protección de los mutualistas. Un claro ejemplo de ello es el reconocimiento del derecho a la asistencia sanitaria del sistema nacional de salud, sin necesidad de suscribir un convenio especial.

\footnotetext{
${ }^{27}$ El mencionado Decreto desarrolla la Ley de 29 de diciembre de 1987 , en lo que concierne a las trabajadoras autónomas y la Ley 11 diciembre de 1990, n 379, en el caso de las profesionales liberales [http://www.inps.it/portale/default.aspx?sID=\%3B0\%3B5673\%3B5689\%3B\&lastMenu=5689\&iMenu=1 \&iNodo=5689\&p4=2]

${ }^{28}$ MISSOC [ec.europa.eu/social/mainjsp?cat1d=1102\&langld=en\&intPageld=2391]

e-Revista Internacional de la Protección Social, ISNN 2445-3269. 2017, $\quad$ Vol. $\quad$ II, $\quad \mathrm{N}^{\mathrm{o}} \quad 2$ http://dx.doi.org/10.12795/e-RIPS.2017.i02.11.

Página 167
} 
Por otro lado, las reformas acometidas a partir de 2013, en aplicación de la Ley 27/2011, al establecer coberturas mínimas, pueden provocar el efecto contrario y forzar la incorporación al RETA de los nuevos profesionales colegiados, quienes- como consecuencia de la subida de las cuotas a satisfacer a la Mutualidad correspondiente-, pueden preferir su encuadramiento en el sistema público de Seguridad Social, que ofrece mejores prestaciones y mayores garantías de pervivencia.

En efecto, en lo que se refiere específicamente a la prestación por maternidad, mientras que las trabajadoras autónomas incluidas en el RETA tienen derecho a la prestación por maternidad en los mismos términos y condiciones que los trabajadores por cuenta ajena, incluido el derecho de cesión a favor del otro progenitor, las profesionales colegiadas han tenido hasta ahora un gran déficit de cobertura en este ámbito.

A partir de la Ley de Igualdad, dicha laguna se cubrió reconociendo al otro progenitor un derecho originario a la prestación (art. 3.4 del RD 295/2009). Ahora bien, en aplicación de la Ley 27/2011 (DA 46 ), las Mutualidades de previsión social, para mantener su condición de "alternativas" al RETA, se han visto obligadas a reconocer a sus mutualistas protección frente a determinadas contingencias, entre las que se encuentra la maternidad. Con la inclusión en su ámbito de cobertura de la prestación por maternidad, aunque se abonen en un único pago, desaparece el presupuesto del derecho atribuido al otro progenitor ex art. 3.4 del RD 295/2009. Así lo están declarando los tribunales de suplicación, por entender que la finalidad de dicho pago es la misma que cumple el subsidio de maternidad del sistema de la Seguridad Social, aun con una duración superior y un modo de pago diferente, y, sobre todo, teniendo en cuenta que estos trabajadores tienen abierta la posibilidad de darse de alta en el Sistema de Seguridad Social. 